Original

\title{
Altered expression of mitochondrial antioxidants in oral squamous cell carcinoma
}

\author{
Sumita Banerjee1), Saikat Mukherjee2), Sanjib Mitra³), and Pallav Singhal4) \\ 1)Department of Oral Pathology, Dental College, Regional Institute of Medical Sciences, Imphal, India \\ 2)Department of Biochemistry, Manipur University, Imphal, India \\ 3)Department of Oral Pathology, Burdwan Dental College and Hospital, Burdwan, India \\ 4)Department of Oral Pathology, Sarjug Dental College and Mata R. Devi Hospital, Darbhanga, India
}

(Received September 6, 2016; Accepted November 25, 2016)

\begin{abstract}
Reactive oxygen species, if produced in excess by oxidative phosphorylation, contributes to mitochondrial DNA damage and progressive respiratory chain dysfunction, leading to various diseases including carcinogenesis. Mitochondria are susceptible to oxidative stress (OS) owing to lack of introns, protective histones, and DNA repair enzymes. However, mitochondria are protected from OS by numerous antioxidants such as superoxide dismutase 2 (SOD2), catalase, glutaredoxin 2 (GLRX2), reduced glutathione (GSH), glutathione peroxidase (GPX), and thioredoxin 2 (TXN2). To obtain insights regarding expression of these mitochondrial antioxidants in oral squamous cell carcinoma (OSCC), we performed qualitative and quantitative estimations of key molecular players of mitochondrial antioxidants during various stages of OSCC by immunoblotting with specific antibodies against antioxidant enzymes and/or biochemical assays. Different mitochondrial antioxidants varied in their expression levels as OSCC progressed. The levels of GPX1, GPX4, and catalase reduced with progression of OSCC. However, GLRX2, PXR3, TXN2, and reduced GSH gradually increased. Expression of SOD2 decreased initially in Stages II and III of OSCC but increased in Stage IV. In conclusion, our findings indicate a complex interplay
\end{abstract}

Correspondence to Dr. Saikat Mukherjee, Department of Biochemistry, Manipur University, Imphal, Manipur 795003, India

E-mail: mukherjee.saikat7@gmail.com

doi.org/10.2334/josnusd.16-0655

DN/JST.JSTAGE/josnusd/16-0655 of various mitochondrial antioxidants in different stages of OSCC, and further insights regarding these molecular players can help us better understand the pathogenesis of OSCC in context of mitochondrial redox status.

Keywords: antioxidants; catalase; glutathione; glutathione peroxidase; mitochondrial superoxide dismutase 2; oral squamous cell carcinoma.

\section{Introduction}

Cancer is a multifactorial disorder, and reactive oxygen species (ROS) plays a vital role in its pathogenesis (1). Mitochondria are a significant source of ROS generation, which includes free radicals, and peroxides are produced as by-products of oxidative phosphorylation $(2,3)$. In addition, mitochondrial DNA is ten times more susceptible to oxidative stress (OS) than nuclear DNA owing to lack of protective histones, introns, and DNA repair systems $(4,5)$ and mitochondrial DNA mutations gradually lead to carcinogenesis (6-8). Moreover, certain physiodynamic alterations of mitochondria occur under OS, which enable tumor cells to react abnormally, showing the Warburg effect, and finally escape the apoptotic process, leading to uncontrolled cellular growth (9). Both exogenous and endogenous antioxidant systems maintain a well-coordinated balance in redox homeostasis within the mitochondria.

Various antioxidants function to regulate ROS and the redox-sensitive pathway in the mitochondria such as manganese superoxide dismutase (MnSOD or SOD2), 
Table 1 Details of patients participating in the study

\begin{tabular}{|c|c|c|}
\hline & & Number of patients \\
\hline \multirow[t]{4}{*}{ Habitual pattern } & Tobacco smoking & 7 \\
\hline & Tobacco chewing & 5 \\
\hline & Smoking + Chewing & 8 \\
\hline & Smoking + Alcohol & 10 \\
\hline Duration of habit (years) & $0-5$ & 4 \\
\hline \multirow[t]{3}{*}{ Displayed in class interval } & $6-10$ & 8 \\
\hline & $11-15$ & 6 \\
\hline & $\geq 15$ & 10 \\
\hline \multirow{3}{*}{$\begin{array}{l}\text { Frequency of habit in case of tobacco smoking } \\
\text { or chewing (no/day) } \\
\text { Displayed in class interval }\end{array}$} & $0-5$ & 9 \\
\hline & $6-10$ & 15 \\
\hline & $11-15$ & 6 \\
\hline Amount of alcohol intake (g/day)* & $10-20 \mathrm{gm}$ & 3 \\
\hline \multirow{2}{*}{ Displayed in class interval } & $21-50 \mathrm{gm}$ & 5 \\
\hline & $>51 \mathrm{gm}$ & 2 \\
\hline \multirow[t]{7}{*}{ Primary site of the lesion } & Floor of the mouth & 6 \\
\hline & Lateral border of tongue & 3 \\
\hline & Ventral surface of tongue & 1 \\
\hline & Buccal mucosa & 10 \\
\hline & Hard palate & 1 \\
\hline & Upper alveolus & 3 \\
\hline & Retromolar area & 6 \\
\hline \multirow[t]{3}{*}{ TNM staging } & Stage II & 12 \\
\hline & Stage III & 7 \\
\hline & Stage IV & 11 \\
\hline
\end{tabular}

mitochondrial glutaredoxin (GLRX2), reduced glutathione (GSH), glutathione peroxidase (GPX), catalase, and thioredoxin 2 (TXN2) systems (10). Several studies have analyzed alterations in the cytosolic level of various cellular antioxidants in the progression of several cancers including oral cancer $(2,11,12)$. However, estimation of these antioxidants in mitochondria at different stages of oral squamous cell carcinoma (OSCC) has not been explored yet. Here, we compared the levels of MnSOD or SOD2, mitochondrial GLRX2, GPX1 and GPX4, reduced GSH, catalase, and the TXN2 system comprising peroxiredoxin 3 (PRX3) and TXN2 in mitochondria of OSCC patients at different TNM stages. Moreover, we compared differences in mitochondrial lipid peroxidation between oral cancer and control groups by estimating the levels of thiobarbituric acid reactive substrates generated during peroxidation of phospholipids.

\section{Patient selection}

\section{Materials and Methods}

In total, 30 cases ( 25 men and 5 women) of newly diagnosed surgical biopsy specimens of OSCC were obtained from the outpatient department and included in the study (Group I). The patients' age ranged 25-50 years.
Histologically, 10 cases were well differentiated, 14 were moderately differentiated, and 6 were poorly differentiated. Clinically, the OSCC cases were categorized into Stage II/III/IV using the tumor, node, and metastasis (TNM) staging system (Table 1). Overall, 20 age- and sex-matched volunteers with tobacco chewing habits but without any present antioxidant medication, any local or systemic infection, and any visible oral lesions were included in the control group (Group II).

The study was approved by the Institutional Ethical Committee, Burdwan Dental College (Memo No: BDCH/164, ID No AC/11/EC/BDCH/2012, Protocol No: 0029/13/P version 9) in the year 2013, and written informed consent forms were obtained from all participants.

The exclusion criteria included patients with prior history, diagnosis, or treatment of cancer with other organ/system, presence of neoplasms at other anatomical site/system at the time of diagnosis of the primary OSCC, and past history or treatment of OSCC. Demographic details, type of habit, frequency, and duration of the habit of the study and control groups were collected in a detailed questionnaire as represented in Table 1. 
Table 2 Relative expression levels of SOD2 in various patient samples as quantified by Image J after normalizing to the loading control mitochondrial marker VDAC1

\begin{tabular}{lllll}
\hline Patient type & No. of patients & $\begin{array}{l}\text { Mean of relative } \\
\text { expression level }\end{array}$ & $\begin{array}{l}\text { Standard deviation of } \\
\text { relative expression level }\end{array}$ & $\begin{array}{l}\text { Variance of relative } \\
\text { expression level }\end{array}$ \\
\hline Control (Group 2) & 20 & 98.5 & 0.87 & 0.765 \\
Stage II & 12 & 46.16 & 0.88 & 0.778 \\
Stage III & 7 & 16.55 & 0.48 & 0.229 \\
Stage IV & 11 & 72.70 & 1.29 & 1.687 \\
\hline
\end{tabular}

\section{Tissue sample collection and preparation of mitochondria}

Surgically resected tumor tissues were immersed in an isotonic homogenization buffer. Mitochondria were isolated by differential centrifugation (Beckman Coulter, Indianapolis, IN, USA), as described (13). The purity of mitochondrial preparation was checked by immunoblotting the crude homogenate and the mitochondrial fraction with various protein markers specific for cytosol ( $\alpha$-tubulin), nucleus (PCNA), endoplasmic reticulum (calreticulin and calnexin), and mitochondria (VDAC1). On the other hand, control group samples were collected using vestibuloplasty.

\section{Measurement of reduced glutathione concentration in mitochondria}

All chemicals were purchased from Sigma-Aldrich (Bangalore, India) unless otherwise mentioned. The levels of oxidized glutathione (GSSG) and reduced GSH were measured using Ellman's method (14). GSH reduces 5, 5'-dithiol (2-nitrobenzoic acid) (DTNB) to 5-thio-2-nitrobenzoic acid (TNB) and gets converted into GSSG. In a coupled reaction, GSSG is reduced by glutathione reductase and NADPH to reduced GSH. TNB was spectrophotometrically measured at $412 \mathrm{~nm}$.

\section{Catalase activity assay}

The catalase activity of the mitochondrial extract was determined at $25^{\circ} \mathrm{C}(15)$. The enzyme reacts with methanol in the presence of hydrogen peroxide forming formaldehyde that reacts with 4-amino3-hydrazine-5-mercapto-1,2,24-triazole (Purpald). Upon oxidation, it changes to purple in color, which was read in UltraViolet-Visible (UV-VIS) spectrophotometer (Eppendorf India Ltd., Kolkata, India) at $540 \mathrm{~nm}$.

\section{Measurement of mitochondrial lipid peroxide content}

The mitochondrial lipid peroxide content was measured as described by Ogura et al (16). The breakdown products of mitochondrial lipid peroxide react with thiobarbituric acid (Sigma-Aldrich), and the resultant chromogen was spectrophotometrically detected at $532.5 \mathrm{~nm}$.

\section{Western blotting}

All chemicals were obtained from Sigma-Aldrich unless mentioned otherwise. Antibodies were purchased either from Sigma-Aldrich or Abcam (Abcam, Cambridge, MA, USA). In brief, $100 \mu \mathrm{g}$ of mitochondrial total protein was separated on 10\% Tris-Glycine SDS-PAGE using Mini-Protean polyacrylamide gel electrophoresis (Bio-Rad Laboratories Inc., Hercules, CA, USA), blotted onto nitrocellulose membrane (Amersham Pharmacia Biotech/GE Healthcare, Kolkata, India), and probed with polyclonal rabbit anti- $\alpha$-tubulin, anti-PCNA, anticalreticulin, anti-calnexin, anti-SOD2, anti-catalase, anti-GLRH2, anti-PRX3, anti-TXN2, anti-GPX1, and anti-GPX4 antibodies at 1:2,000 dilutions for $2 \mathrm{~h}$ at $4^{\circ} \mathrm{C}$ in parallel sets and then with mouse anti-rabbit IgG secondary antibody conjugated to horseradish peroxidase (HRP) at 1:10,000 dilutions for $1 \mathrm{~h}$ at room temperature. Chemiluminescence detection of protein bands was conducted using an ECL kit (Amersham Pharmacia Biotech/GE HealthCare). For loading control, rabbit polyclonal anti voltage-dependent anion channel 1 (VDAC1) antibody was used. Relative quantifications of SOD2 protein bands with respect to loading controls were performed using Image $\mathrm{J}$ software on the western blot films (Table 2).

\section{Statistical analysis}

Statistical analysis was conducted using the statistical package for social sciences (SPSS version 15.0; SPSS, Chicago, IL, USA) statistical analysis software. Comparisons of biochemical parameters between case and control groups were made using independent student $t$-test, and the values were expressed as mean $\pm \mathrm{SD}$. One-way analysis of variance (ANOVA) test was used to compare the parameters in different TNM staging. $P$ values $<0.05$ were considered statistically significant. Tukey's honest significant difference (HSD) test was used to reveal statistical differences in mean values between different groups, and $P<0.05$ was considered significant. 
Table 3 Analysis of concentration/activities of reduced glutathione, lipid peroxides, and catalase in different experimental and control groups

\begin{tabular}{|c|c|c|c|c|c|}
\hline \multirow[t]{2}{*}{ Test molecules } & \multicolumn{5}{|c|}{ Concentration of experimental molecules/Activities of enzymes (Mean \pm SD) } \\
\hline & $\begin{array}{l}\text { Control } \\
n=20\end{array}$ & $\begin{array}{l}\text { Stage I } \\
n=30\end{array}$ & $\begin{array}{l}\text { Stage II } \\
n=12\end{array}$ & $\begin{array}{l}\text { Stage III } \\
n=7\end{array}$ & $\begin{array}{l}\text { Stage IV } \\
n=11\end{array}$ \\
\hline Reduced glutathione & $11.1 \pm 2.38(\mathrm{mM})$ & $15.2 \pm 3.87(\mathrm{mM})$ & $13.2 \pm 2.36(\mathrm{mM})$ & $15.3 \pm 2.63(\mathrm{mM})$ & $17.2 \pm 3.25(\mathrm{mM})$ \\
\hline Lipid peroxide & $\begin{array}{l}1.49 \pm 0.189 \\
(\text { nmole } / \mathrm{mg} \text { of protein) }\end{array}$ & $\begin{array}{l}0.792 \pm 7.491 \\
(\text { nmole/mg of protein) }\end{array}$ & $\begin{array}{l}0.841 \pm 8.25 \\
(\text { nmole } / \mathrm{mg} \text { of protein })\end{array}$ & $\begin{array}{l}0.743 \pm 3.30 \\
(\text { nmole } / \mathrm{mg} \text { of protein })\end{array}$ & $\begin{array}{l}0.659 \pm 5.33 \\
(\text { nmole } / \mathrm{mg} \text { of protein })\end{array}$ \\
\hline Catalase & $6.410 \pm 0.285 \mathrm{mg} / \mathrm{min}$ & $2.66 \pm 0.314 \mathrm{mg} / \mathrm{min}$ & $3.13 \pm 0.521 \mathrm{mg} / \mathrm{min}$ & $2.2 \pm 0.321 \mathrm{mg} / \mathrm{min}$ & $2.0 \pm 0.209 \mathrm{mg} / \mathrm{min}$ \\
\hline
\end{tabular}

Table 4 ANOVA test for concentration/activity levels of reduced glutathione, lipid peroxide, and catalase as derived from Table 3

\begin{tabular}{lllllll}
\hline & Sum of squares & Degrees of freedom $(\mathrm{df})$ & Mean of square & $\mathrm{F}$ & $P$ & Significance \\
\hline Reduced glutathione & 225.5 & 79 & 56.38 & 12.937 & 0.000 & $\mathrm{~S}$ \\
Lipid peroxide & 175.3 & 79 & 85.38 & 0.087 & 0.986 & NS \\
Catalase expression & 314.5 & 79 & 43.58 & 5.078 & 0.000 & S \\
\hline
\end{tabular}

$\mathrm{S}=$ statistically significant $(P<0.05), \mathrm{NS}=$ statistically nonsignificant $(P>0.05)$.

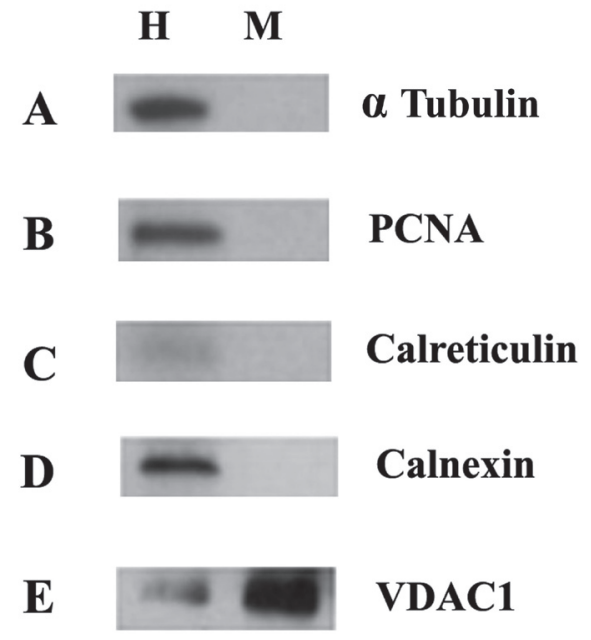

Fig. 1 Representative immunoblots using different protein markers to check the purity of the mitochondria isolated from oral tissue. " $\mathrm{H}$ " indicates total tissue homogenate, and " $\mathrm{M}$ " indicates mitochondrial fraction isolated by differential centrifugation. (A) $\alpha$-tubulin, cytosolic marker; (B) PCNA, nuclear marker; (C) calreticulin, endoplasmic reticulum marker; (D) calnexin, endoplasmic reticulum marker; (E) VDAC1, mitochondrial marker.

\section{Results}

Mitochondria obtained after differential centrifugation were identified as pure without any cytosolic or other organellar contamination (Fig. 1). The extent of lipid peroxidation and the concentration/activity levels of various mitochondrial antioxidants were compared during various clinical stages of OSCC (Stages I, II, III, and IV) and control groups by either biochemical activity assays or western blotting of mitochondrial extracts of tissue samples. Table 3 enlists the concentration/activity levels of reduced GSH, catalase, and lipid peroxide, as measured using the biochemical approach. The concentrations of reduced GSH increased during different stages of OSCC, as determined in OSCC patients from Stage I $(15.2 \pm 3.87 \mathrm{mM})$, Stage II $(13.2 \pm 2.36 \mathrm{mM})$, Stage III $(15.3 \pm 2.63 \mathrm{mM})$, and Stage IV $(17.2 \pm 3.25 \mathrm{mM})$ in comparison with control or Group II participants (11.1 $\pm 2.38 \mathrm{mM}$ ). Moreover, the expression of lipid peroxide reduced in OSCC patients from Stage I $(0.792 \pm 7.491$ nmole/mg of protein), Stage II $(0.841 \pm 8.25 \mathrm{nmole} / \mathrm{mg}$ of protein), Stage III ( $0.743 \pm 3.30 \mathrm{nmole} / \mathrm{mg}$ of protein), and Stage IV $(0.659 \pm 5.33 \mathrm{nmole} / \mathrm{mg}$ of protein $)$ in comparison with control or Group II participants (1.49 \pm $0.189 \mathrm{nmole} / \mathrm{mg}$ of protein). Similarly, catalase activity reduced in Stage I $(2.66 \pm 0.314 \mathrm{U} / \mathrm{mg} / \mathrm{min})$, Stage II $(3.13 \pm 0.521 \mathrm{U} / \mathrm{mg} / \mathrm{min})$, Stage III $(2.2 \pm 0.321 \mathrm{U} / \mathrm{mg} /$ $\mathrm{min})$, and Stage IV $(2.0 \pm 0.209 \mathrm{U} / \mathrm{mg} / \mathrm{min})$ in comparison with Group II $(6.410 \pm 0.285 \mathrm{U} / \mathrm{mg} / \mathrm{min})$. In order to understand the differences between groups, means, and the alterations during different stages of cancer, ANOVA was applied on the expression levels of reduced GSH, lipid peroxide, and catalase (Table 3) by putting the variables in an ANOVA chart using SPSS (version 15). The results of ANOVA are summarized in Table 4, detailing the statistical values of various parameters such as sum of squares, degrees of freedom, mean of squares, F, and $P$ values, with $P$ values being the most important parameter in ANOVA; $P<0.05$ is considered statistically significant (denoted by "S" in Table 4), whereas $P>$ 0.05 is considered statistically nonsignificant (denoted by "NS" in Table 4). According to the ANOVA analysis, 
Table 5 Analysis of significant/nonsignificant changes in reduced glutathione concentrations in the control group and patients with different stages of OSCC by post-hoc Tukey's HSD test

\begin{tabular}{|c|c|c|c|c|c|c|}
\hline $\mathrm{SN}$ & Comparison & Mean difference & $\mathrm{q}$ & $95 \% \mathrm{CI}$ & $P$ & Significance \\
\hline 1. & Control vs Stage I & 4.1000 & -3.86 & 1.5477 to 6.6523 & 0.0002 & $\mathrm{~S}$ \\
\hline 2. & Control vs Stage II & 2.1000 & -1.58 & 1.1284 to 5.3284 & 0.3709 & NS \\
\hline 3. & Control vs Stage III & 4.2000 & -2.9 & 0.3173 to 8.0827 & 0.0274 & $\mathrm{~S}$ \\
\hline 4. & Control vs Stage IV & 6.1000 & -4.52 & 2.7811 to 9.4189 & 0.0000 & $\mathrm{~S}$ \\
\hline 5. & Group I vs Stage II & 2.0000 & -1.73 & 5.0199 to 1.0199 & 0.3526 & NS \\
\hline 6. & Group I vs Stage III & 0.1000 & -0.08 & 3.6112 to 3.8112 & 1.0020 & NS \\
\hline 7. & Group I vs Stage IV & 2.0000 & -1.71 & 1.1164 to 5.1164 & 0.3848 & NS \\
\hline 8. & Stage II vs Stage III & 2.10002 & -1.22 & 1.0490 to 1.0390 & 0.03621 & $\mathrm{~S}$ \\
\hline 9. & Stage II vs stage IV & 4.0000 & -2.26 & 0.3094 to 7.6906 & 0.0270 & $\mathrm{~S}$ \\
\hline 10. & Stage III vs Stage IV & 1.9000 & -1.04 & 2.3748 to 6.1748 & 0.7267 & NS \\
\hline
\end{tabular}

$\mathrm{S}=$ statistically significant $(P<0.05)$, NS $=$ statistically nonsignificant $(P>0.05)$.

Table 6 Analysis of significant/nonsignificant changes of catalase activity in the control group and patients with different stages of OSCC by post-hoc Tukey's HSD test

\begin{tabular}{|c|c|c|c|c|c|c|}
\hline SN & Comparison & Mean difference & $\mathrm{q}$ & $95 \% \mathrm{CI}$ & $P$ & Significance \\
\hline 1. & Control vs Stage I & 0.4700 & -0.40 & 0.1497 to 0.7903 & 0.0010 & $\mathrm{~S}$ \\
\hline 2. & Control vs Stage II & 3.2800 & -2.47 & 3.6225 to -2.9375 & 0.2589 & NS \\
\hline 3. & Control vs Stage III & -0.6600 & 0.56 & -0.9906 to -0.3294 & 0.0000 & S \\
\hline 4. & Control vs Stage IV & -0.9300 & 0.54 & 1.3761 to -0.4839 & 0.0000 & S \\
\hline 5. & Group I vs Stage II & -1.1300 & 0.72 & -1.5215 to -0.7385 & 0.0000 & S \\
\hline 6. & Group I vs Stage III & 0.4600 & 0.37 & 0.8537 to -0.0663 & 0.0138 & S \\
\hline 7. & Group I vs Stage IV & -0.4600 & 0.36 & -0.8906 to -0.7294 & 0.0384 & S \\
\hline 8. & Stage II vs Stage III & 4.2100 & -2.91 & 4.6219 to -3.7981 & 0.5442 & NS \\
\hline 9. & Stage II vs stage IV & 3.2000 & -2.8 & 0.0173 to 0.0827 & 0.0164 & S \\
\hline 10. & Stage III vs Stage IV & -0.2000 & 0.11 & 0.0535 to 0.0335 & 0.0023 & $\mathrm{~S}$ \\
\hline
\end{tabular}

$\mathrm{S}=$ statistically significant $(P<0.05), \mathrm{NS}=$ statistically nonsignificant $(P>0.05)$.

the concentration/activity of reduced GSH and catalase induced significant changes during $\operatorname{OSCC}(P=0.000)$. On the other hand, lipid peroxide showed nonsignificant changes in OSCC $(P=0.986)$. To analyze the correlation between different groups, post-hoc Tukey's HSD test was applied; the test was applied only for reduced GSH and catalase because the concentration/activity levels of these two enzymes alone were found statistically significant in ANOVA. All statistical parameters analyzed in Table 5 (representing analysis for reduced GSH) and Table 6 (representing analysis for catalase) was outcomes of post-hoc Tukey's HSD test. Tables 5 and 6 present individual comparisons between all of the different study groups to determine if any study group showed any significant changes in reduced GSH and catalase expression in comparison with each other. In addition, Tables 5 and 6 also enlist the outcomes of posthoc Tukey's HSD for various statistical parameters such as mean differences, $\mathrm{q}, 95 \% \mathrm{CI}$, and $P$ values, with $P$ values being the most important; $P$ values $<0.05$ indicate statistically significant (denoted by "S"), whereas $P$ value $>0.05$ were considered statistically insignificant (denoted by "NS"). For reduced GSH, significant changes were observed between the control group and the OSCC
Stage I, between the control group participants and Stage III patients, and between the control group participants and Stage IV patients. Intra group comparisons of OSCC patients revealed statistically significant changes between Stage II and Stage III patients and between the Stage II and Stage IV patients. However, Stage III and Stage IV patients revealed no statistically significant changes in reduced GSH levels. There is a significant change in reduced GSH level in Stage III in comparison to other cancer stages which indicate the vital role of this enzyme in cancer progression; however, additional studies are warranted to investigate this further.

On performing similar comparisons for catalase expression among different groups, statistically significant changes were noted among the control group participants and Stage I OSCC patients, controls and Stage III patients, and controls and Stage IV patients. Moreover, intra group comparisons for various stages for OSCC revealed statistically significant changes between Stage II and Stage IV patients as well as between Stage III and Stage IV patients. However, Stage II and Stage III patients revealed no significant differences. These findings indicate that alterations in catalase expression from Stage III to Stage IV might have a pivotal role in 
$\mathbf{A}$

B

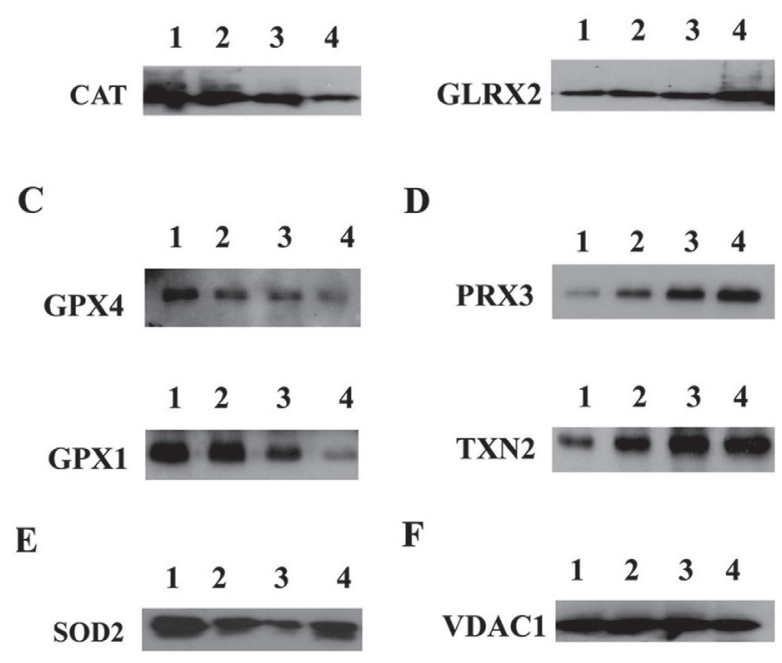

Fig. 2 Representative immunoblots showing expression levels of various mitochondrial antioxidants in different stages of oral squamous cell carcinoma. (A) catalase, CAT; (B) glutaredoxin 2, GLRX2; (C) glutathione peroxidase 1 (GPX1) and glutathione peroxidase 4 (GPX4); (D) peroxiredoxin 3 (PRX3) and thioredoxin 2 (TXN2); (E) superoxide dismutase 2 (SOD2); (F) voltage-dependent anion channel 1 (VDAC1) as a housekeeping protein in mitochondrial immunoblot analysis. Lane 1: control group; Lane 2: OSCC stage II; Lane 3: OSCC Stage III; Lane 4: OSCC Stage IV.

the progression of OSCC, which must be investigated in future studies.

Besides biochemical tests, immunoblotting of patient tissue samples was performed with antibodies against various antioxidants. Significant decrease in catalase expression was noted in immunoblotting (Fig. 2A). Moreover, GPX1 and GPX4 levels decreased with progression of OSCC (Fig. 2C). On the other hand, GLRX2, PRX3, and TXN2 showed increased expression in OSCC (Fig. 2B, 2D). Furthermore, mitochondrial SOD2 showed reduced expression as OSCC progressed (Stages II and III); however, at advanced stages (Stage IV), SOD2 expression increased (Fig. 2E). Quantification of SOD2 protein bands using Image $J$ showed a relative decrease of SOD2 in Stage II and Stage III patients but an increase in Stage IV patients in comparison with the control group (Table 5). Although the control group (Group 2) showed a mean relative SOD2 expression level of 98.5 when western blot films were scanned using Image J, Stage II and Stage III patients showed a gradual decrease, with mean relative SOD2 expression levels of 46.16 and 16.15, respectively. However, Stage IV patients showed a significant increase in the mean relative SOD2 expression level (72.70). VDAC1 was taken as a loading control in mitochondrial immunoblot analysis, and its levels remained unaffected in both the control and OSCC groups (Fig. 2F).

\section{Discussion}

Our study presents an interesting profiling of various mitochondrial antioxidants in the mitochondrial redox landscape and its alteration during oral carcinogenesis. SOD2 is known to be a key controller of cell proliferation (17), but its role in carcinogenesis remains controversial. Depending on its concentration and specific contexts, it modulates the growth of cancer cells (18). In this study, SOD2 levels decreased in the early stages of OSCC (Stages II and III) but increased significantly in Stage IV. However, Liu et al. reported a constant increase of SOD2 levels with ascending grades of tongue squamous cell carcinoma (19). Most previous studies related to SOD2 levels in OSCC have been conducted in cell lines, and only few in vivo studies have been conducted. In this study, the altered levels of SOD2 may be attributed to the self-defense mechanism of the immune system and the biphasic control of SOD2 on cancer cells (18). On the other hand, the sudden increase of SOD2 in Stage IV OSCC compared to Stage III (Fig. 2E) supports the findings of Liu et al. (19) who also reported increased levels of SOD2 in metastatic tumors.

GRX2, another major antioxidant of mitochondria, blocks cytochrome c release and caspase activation, preventing apoptotic cell death under OS (20). It facilitates tumor angiogenesis, thus promoting additional cell proliferation (21). A gradual increase in GLRX2 levels with progressive stages of OSCC in comparison with the control group, as observed in the present study, support its antiapoptotic function. Both GPX1 and GPX4 have antitumorigenic properties (10). In this study, both of these enzymes significantly decreased with progressive stages of OSCC, which is similar to pancreatic and breast cancer $(22,23)$. We also assessed the components of the TXN2 system, a prime target for anticancer therapy nowadays (10), and found an overall increase in these components (i.e., TXN2 and PRX3) with progression of OSCC. Tumor cells are more dependent on the TXN2 system owing to their constant demand of DNA; thus, suppression of the TXN2 system can be a promising strategy for oral cancer therapy. Such an example of targeting TXN2 was done in treatment of multiple myelomas (24). Reduced GSH plays a major role in maintaining intracellular redox balance. In various cancers, GSH levels are increased, making them more resistant to ROS $(25,26)$. Similar findings were noted for mitochondrial GSH in the present study as well. For lipid peroxide levels, a decrease in 
mean values were noted in both controls and OSCC patients, which, however, was not statistically significant (Table 2). Phospholipids are potent substrates for peroxidation in the mitochondrial membrane of OSCC tissues, and a decline in lipid peroxide levels indicates loss of phospholipids. Membranes of cancer cells have more cholesterol than phospholipids as cholesterol decreases the susceptibility of cancer cells to ROS attack (27). The decrease in mean values of lipid peroxides in our cancer samples can indirectly signify the loss of phospholipids in the membrane, even if the decrease may not be statistically significant owing to small sample size. Catalase has a protective role in cancer progression and invasion (28). The decrease in catalase levels with progression of cancer stages signifies the loss of the catalase-mediated protective mechanism in cancer microenvironment. Our preliminary findings establish that mitochondrial antioxidants might be correlated with progression in OSCC. It would be worth studying if there is any molecular interplay of mitochondrial and cytosolic antioxidants in the context of OSCC.

\section{Acknowledgments}

The authors wish to thank all participants who joined the study. The authors also express their sincerest thanks to Dr. Aswini Y. Balappanavar, Maulana Azad Institute of Dental Sciences, New Delhi, for the statistical analysis and Professor Nandini D.B., Department of Oral Pathology, Regional Institute of Medical Sciences, Imphal, for the valuable suggestions.

\section{Conflict of interest}

The authors have no conflict of interest to declare.

\section{References}

1. Srivastava KC, Austin RD, Shrivastava D (2016) Evaluation of oxidant-antioxidant status in tissue samples in oral cancer: a case control study. Dent Res J 13, 181-187.

2. Archer SL, Gomberg-Maitland M, Maitland ML, Rich S, Garcia JG, Weir EK (2008) Mitochondrial metabolism, redox signaling and fusion: a mitochondria-ROS-HIF- $1 \alpha-\mathrm{Kv} 1.5$ $\mathrm{O}_{2}$-sensing pathway at the intersection of pulmonary hypertension and cancer. Am J Physiol Heart Circ Physiol 294, H570-578.

3. Addabbo F, Montagnani M, Goligorsky MS (2009) Mitochondria and reactive oxygen species. Hypertension 53, 885-892.

4. Yu YP, Yu G, Tseng G, Cieply K, Nelson J, Defrances M et al. (2007) Glutathione peroxidase 3, deleted or methylated in prostate cancer, suppresses prostate cancer growth and metastasis. Cancer Res 67, 8043-8050.

5. Yoo DG, Song YJ, Cho EJ, Lee SK, Park JB, Yu JH et al. (2008) Alteration of APEl/ref-l expression in non-small cell lung cancer: the implications of impaired extracellular superoxide dismutase and catalase antioxidant systems. Lung Cancer 60, 277-284.

6. Burgart LJ, Zheng J, Shu Q, Strickler JG, Shibata D (1995) Somatic mitochondrial mutation in gastric cancer. Am J Pathol 147, 1105-1111.

7. Alonso A, Martin P, Albarran C, Aguilera B, Garcia O, Guzman A et al. (1997) Detection of somatic mutations in the mitochondrial DNA control region of colorectal and gastric tumors by heteroduplex and single-strand conformation analysis. Electrophoresis 18, 682-685.

8. Fliss MS, Usadel H, Caballero OL, Wu L, Buta MR, Eleff SM et al. (2000) Facile detection of mitochondrial DNA mutations in tumors and bodily fluids. Science 287, 2017-2019.

9. Roberts ER, Thomas KJ (2013) The role of mitochondria in the development and progression of lung cancer. Comput Struct Biotechnol J 6, e201303019.

10. Chen Y, Zhang H, Zhou HJ, Ji W, Min W (2016) Mitochondrial redox signaling and tumor progression. Cancers, doi: 10.3390/cancers8040040.

11. Ho JC, Zheng S, Comhair SA, Farver C, Erzurum SC (2001) Differential expression of manganese superoxide dismutase and catalase in lung cancer. Cancer Res 61, 8578-8585.

12. Perše M (2013) Oxidative stress in the pathogenesis of colorectal cancer: cause or consequence? Biomed Res Int, 725710 .

13. Kokoszka JE, Coskun P, Esposito LA, Wallace DC (2001) Increased mitochondrial oxidative stress in the Sod2 (+/-) mouse results in the age-related decline of mitochondrial function culminating in increased apoptosis. Proc Natl Acad Sci U S A 98, 2278-2283.

14. Akerboom TP, Sies H (1981) Assay of glutathione, glutathione disulfide, and glutathione mixed disulfides in biological samples. Methods Enzymol 77, 373-382.

15. Johansson LH, Borg LA (1988) A spectrophotometric method for determination of catalase activity in small tissue samples. Anal Biochem 174, 331-336.

16. Ogura R, Sakanashi T, Nagata O, Sugiyama M, Kajiyama K, Nakagawa T et al. (1987) Assay for lipid peroxide content in mitochondria by the thiobarbituric acid reaction. Kurume Med J 34, 53-58.

17. St Clair DK, Holland JC (1991) Complementary DNA encoding human colon cancer manganese superoxide dismutase and the expression of its gene in human cells. Cancer Res 51, 939-943.

18. Inoue T, Suzuki-Karasaki Y (2013) Mitochondrial superoxide mediates mitochondrial and endoplasmic reticulum dysfunctions in TRAIL-induced apoptosis in Jurkat cells. Free Radic Biol Med 61, 273-284.

19. Liu Z, Li S, Cai Y, Wang A, He Q, Zheng C et al. (2012) Manganese superoxide dismutase induces migration and invasion of tongue squamous cell carcinoma via $\mathrm{H}_{2} \mathrm{O}_{2}$-dependent Snail signaling. Free Radic Biol Med 53, 44-50.

20. Enoksson M, Fernandes AP, Prast S, Lillig CH, Holmgren A, Orrenius S (2005) Overexpression of glutaredoxin 2 attenu- 
ates apoptosis by preventing cytochrome c release. Biochem Biophys Res Commun 327, 774-779.

21. Lillig CH, Lönn ME, Enoksson M, Fernandes AP, Holmgren A (2004) Short interfering RNA-mediated silencing of glutaredoxin 2 increases the sensitivity of HeLa cells toward doxorubicin and phenylarsine oxide. Proc Natl Acad Sci U S A 101, 13227-13232.

22. Liu J, Du J, Zhang Y, Sun W, Smith BJ, Oberley LW et al. (2006) Suppression of the malignant phenotype in pancreatic cancer by overexpression of phospholipid hydroperoxide glutathione peroxidase. Hum Gene Ther 17, 105-116.

23. Cejas P, García-Cabezas MA, Casado E, Belda-Iniesta C, De Castro J, Fresno JA et al. (2007) Phospholipid hydroperoxide glutathione peroxidase (PHGPx) expression is downregulated in poorly differentiated breast invasive ductal carcinoma. Free Radic Res 41, 681-687.
24. Fink EE, Mannava S, Bagati A, Bianchi-Smiraglia A, Nair JR, Moparthy K et al. (2016) Mitochondrial thioredoxin reductase regulates major cytotoxicity pathways of proteasome inhibitors in multiple myeloma cells. Leukemia 30, 104-111.

25. Calvert P, Yao KS, Hamilton TC, O’Dwyer PJ (1998) Clinical studies of reversal of drug resistance based on glutathione. Chem Biol Interact 111-112, 213-224.

26. Sies H (1999) Glutathione and its role in cellular functions. Free Radic Biol Med 27, 916-921.

27. Dianzani MU (1993) Lipid peroxidation and cancer. Crit Rev Oncol Hematol 15, 125-147.

28. Goh J, Enns L, Fatemie S, Hopkins H, Morton J, Pettan-Brewer C et al. (2011) Mitochondrial targeted catalase suppresses invasive breast cancer in mice. BMC Cancer 11, 191. 